Journal of

Accident and

Emergency

Medicine 1994

11, 67-77
Correspondence:

D.F. Heath,

Northern Western

Injury Research

Centre, Clinical

Sciences Building,

Hope Hospital, Eccles

Old Road, Salford M6

$8 \mathrm{HD}$.

\title{
Glucose, insulin and other plasma metabolites shortly after injury
}

\author{
D. F. HEATH
}

North Western Injury Research Centre, Clinical Sciences Building, Hope Hospital, Salford

\section{SUMMARY}

A statistical study was made of measurements within $3 \mathrm{~h}$ of injury on 533 patients grouped by injury severity using ISS. A scoring system was also used that took account of the number of injuries. Widely accepted hypotheses about the development of hyperglycaemia were not supported. There was evidence of inhibition of glucose metabolism ('insulin resistance'), but none for any particular mechanism. The only factor that was closely related within any group to [glucose] ${ }^{\star}$ (plasma glucose concentration) was [lactate], the higher mean value and variance of which after very severe injuries (ISS above 15) could account for much of the higher mean and variance of glucose in this ISS range. At ISS 9-14, up to $1.4 \mathrm{~h}$ after injury, only $4 \%$ of the variance of glucose could be accounted for by any combination of the concentrations of catecholamines, cortisol, lactate and vasopressin, times of measurement after injury and food intake, and injury severity and number of injuries. There was also no dependence on the part of the body injured.

Injury increased the variability of [insulin] less than published statements imply. The increase found was entirely explicable, as expected, by the changes of [glucose] and [adrenaline].

Key words: catecholamines, glucose, hormones, injury, ISS, insulin

\section{INTRODUCTION}

It is well known that plasma glucose concentration, [glucose], rises rapidly and very variably after injury. In brief, the accepted theory of the mechanism is as follows. Hepatic output of glucose is increased by stimulation of hepatic glycogenolysis by adrenaline and glucagon and by gluconeogenesis from lactate,

\footnotetext{
* Concentrations in plasma are denoted by square brackets.
}

released in part from injured tissue and in part by stimulation of muscle glycogenolysis and glycolysis by adrenaline. It is possible that hepatic glycogenolysis is also stimulated by vasopressin, angiotensin II and activation of the sympathetic fibres in the hepatic nerve. Glucose uptake is inhibited by 'insulin resistance' involving cortisol and adrenaline. The increase of [glucose] with injury severity, at least as measured by ISS, is mediated entirely by the effects of injury on [adrenaline]. ${ }^{1}$ For reviews, see Barton ${ }^{2}$ and Douglas \& Shaw. ${ }^{3}$

The above theory holds for the rat. The initial rise in [glucose] can be accounted for by the action of adrenaline, by gluconeogenesis from lactate released from damaged tissue and by adrenalinestimulated glycolysis in muscle. There is no rise in glucose after $24 \mathrm{~h}$ starvation, when glycogen stores are depleted, ${ }^{4}$ insulin resistance develops rapidly ${ }^{5}$ and depends upon increased [corticosterone]. ${ }^{6}$ The rise in [glucagon] probably contributes to the maintenance of hyperglycaemia, ${ }^{7}$ although the [glucagon]/[insulin] ratio is not raised. ${ }^{8}$

If this theory is correct then it should be able to account for the values of [glucose] that are measured in patients following injury. Failure would indicate that this theory is inadequate, however well it is supported by the results of controlled experiments in animals.

Previous studies have not been promising ${ }^{9-12}$ as they rarely accounted for a quarter of the variance of [glucose]. However, each study is open to criticism on one or more of the following counts. (1) Measurements made after treatment and/or several hours after injury were included in the analysis without full investigation of the effects of treatment and time; (2) inappropriate statistical methods were used; (3) the interactions of the various factors were underinvestigated; and (4) the results from patients with a very wide range of severity of injury were lumped together for analysis. The last factor, as shown below, had particularly confusing effects. 
I have attempted a more complete study, confining attention to measurements made within $3 \mathrm{~h}$ of injury, using multivariate analysis, and including some factors not previously considered (i.e. specificity of injury and unconsciousness). In conjunction with ISS a new scale was used, ISS-AIS, which took account of all the injuries. The aim was to explain the variability of the values of [glucose] (Fig. 1) and [insulin] (Fig. 2), which were measured within the fairly narrow bands of injury severity shown. These, suggested by Copes et al., ${ }^{13}$ correspond to minor (ISS 1-3), moderate $(4-8)$, severe $(9-14)$, very severe (16-24) and life-threatening $(25+)$ injuries. Success has been measured by the percentage of the variance within each group that can be accounted for by the various factors.

Two sets of data have been used. The first data set is that shown in Figs 1-4, and consists of all the plasma metabolite concentrations measured in the North Western Injury Research Centre up to mid1990 on normal and injured subjects with the exception of those from patients with injuries with a known tendancy to produce aberrant values, namely adults with burns, ${ }^{14}$ children ${ }^{14}$ and those suffering from an illness at the time of injury. ${ }^{11}$ This set corresponded to those used in most earlier studies, and had to be used if comparisons were to made with those studies. However, this data set was statistically heterogeneous, and a subset in the age range 17-65, with other limitations as described below, was used for more exact analysis.

Multivariate analysis had to be used with care as statistical tests based on it require residuals (the portions of the measurements not accounted for) to be normally distributed. It soon became clear that this was rarely the case. It is usual to neglect nonnormality, on the grounds that the method is 'fairly robust', i.e., the results are little affected by it. Robustness can, however, only be assessed by using simulated data which may or may not reflect the quirkiness of actual measurements. In the case of the data used in this study non-normality mattered, because when residuals were made more normal by transforming the data to logarithms or square roots, the percentage of the variance which was accounted for by theory was always increased, sometimes substantially. Because the aim was to account for as much of the variance as possible
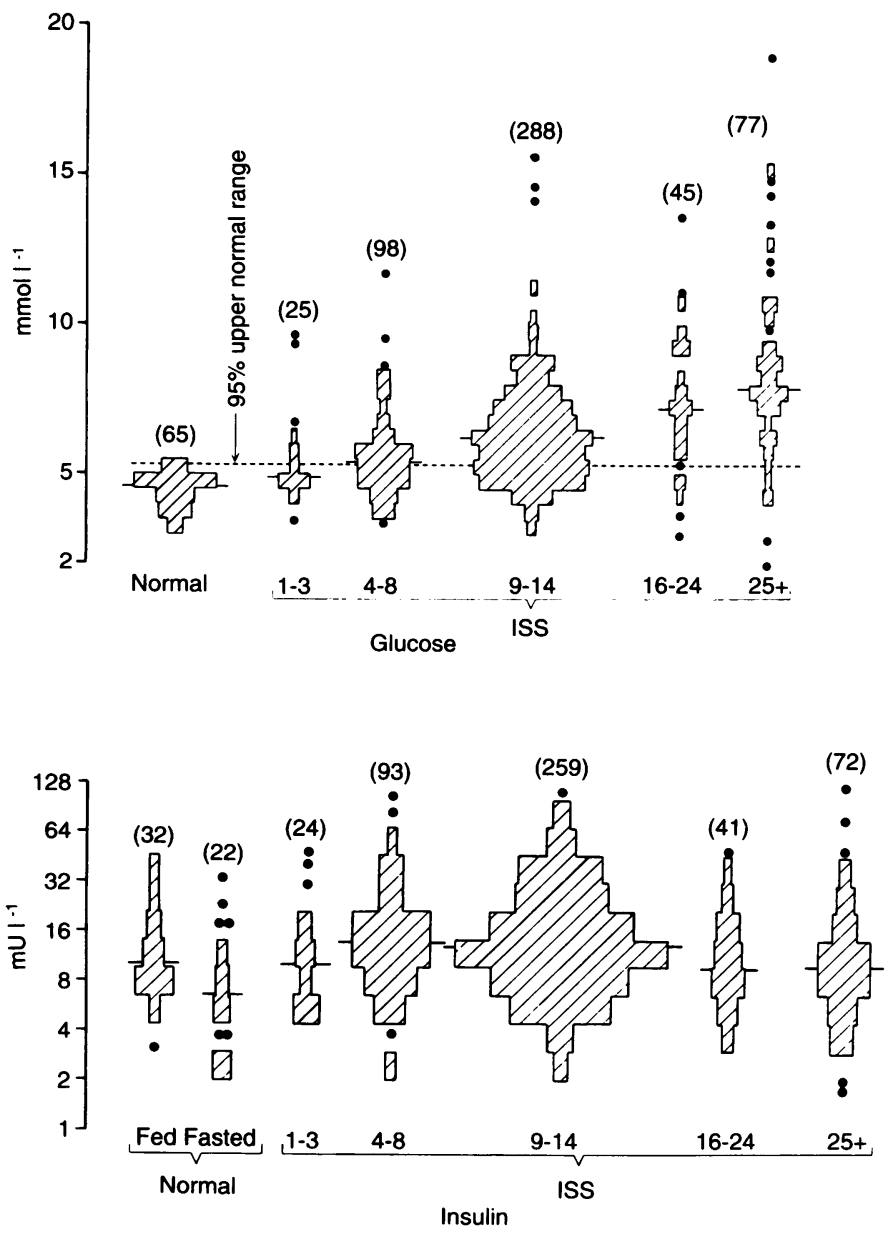

Fig. 1. The distribution of plasma glucose concentrations in normal subjects and 533 patients within $3 \mathrm{~h}$ of injury. The bars represent median values, the dashed line the upper $95 \%$ limit of the normal range. The number in each group is shown in parentheses.

Fig. 2. The distribution of plasma insulin concentrations in normal subjects and 489 patients within $3 \mathrm{~h}$ of injury. The bars represent median values; the number in each group is shown in parentheses. 'Fed' refers to normal subjects studied $2-5 \mathrm{~h}$ after food. 
Glucose and insulin shortly after injury

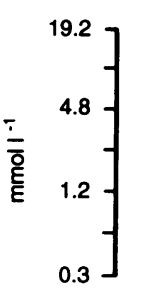

Lacatate

$\left.\begin{array}{cc} & 2.0 \\ \overline{\bar{g}} & 1.0 \\ \bar{\varepsilon} & \\ & 0\end{array}\right]$

NEFA
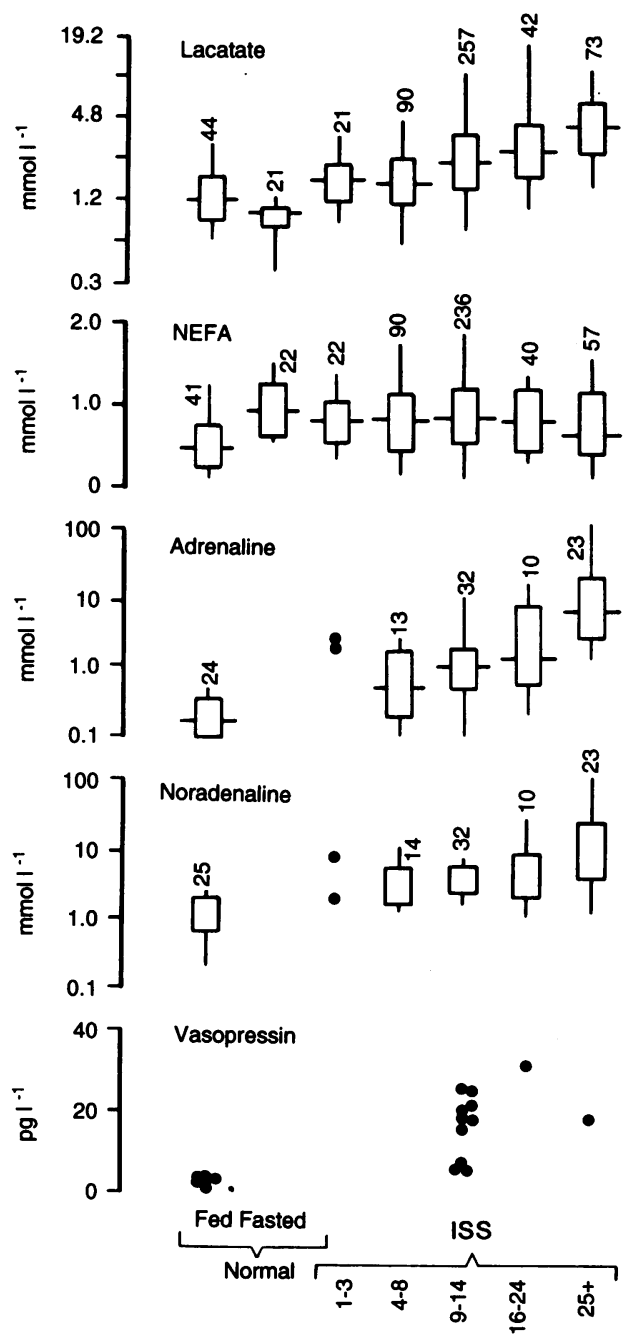

Fig. 3. The concentrations of plasma lactate, NEFA, adrenaline, noradrenaline and vasopressin measured in normal subjects and patients within $3 \mathrm{~h}$ of injury. The upper and lower limits of each box denote the central two thirds of values, the whiskers the range and the bars the medians. Group sizes are shown. Individual values are shown where numbers are very low. 'Fed' refers to subjects $2-5 \mathrm{~h}$ after food.

only the results with the 'best' (most normalizing) transformations are shown, along with the transformations themselves.

\section{SUBJECTS AND METHODS}

\section{Subjects}

There were 533 patients in the main set described previously and 303 in the subset aged between 17 and 65 years. Time after injury was usually known to within 5-15 min, and was recorded to $0.1 \mathrm{~h}$. subjects aged 17-59 years. Most of the data have been published; ${ }^{1,11,14-16}$ but the analysis is entirely new. All work had received approval from the Salford (UK) Area Ethical Committee.

Injury severity

Severity was measured by the 1980 version of AIS, except for brain injuries, for which the 1985 version was used. For most other injuries suffered (few were penetrating) both versions gave the same scores. ${ }^{17}$ Relevant differences are noted.

ISS only takes account of the worst injury in each of the three worst injured body areas. Two AISbased systems that took account of all the injuries were tested. The better, ISS-AIS, is used here, and consists of ISS plus the sum of the scores not used in its calculation. One patient, for example, with leg injuries, had 4 of AIS 3 to one leg and 4 of AIS 2 to the other. The ISS was 9, the square of the highest AIS, but the ISS-AIS was 26 , i.e., $9+9+8-$ namely the ISS plus the sum of the three unused scores of 3 and the 4 of 2 .

\section{Blood collection and measurement of metabolites}

A specimen of each patient's blood was taken for measurement of metabolites shortly after arrival in hospital and usually before treatment, although 38 patients had received analgaesics and 16 Haemaccel. When more than one blood specimen was taken, measurements from the first specimen only have been included in this study. From normal subjects blood was taken after an overnight fast of $10-15 \mathrm{~h}$ (fasted) or $2-5 \mathrm{~h}$ after breakfast or lunch (fed).

At least four compounds were measured on each blood specimen, by methods described in the original papers except for [glucagon], that was measured by a kit (Novo-Nordisk a/s, Bagsvaerd, Denmark), and vasopressin, that was measured by Dr M. Forsling, Guys Hospital, London, UK. The method for measuring [cortisol] was changed in 1978, and only later values have been included. The four values of [adrenaline] that were too low to measure were assigned the value $0.1 \mathrm{nmole}^{-1}$, which was close to the sensitivity of the method.

\section{Data handling and statistical analysis}

Searches and most of the statistics were carried out using a purpose-written database on a PDP 11/34 minicomputer. Some multivariate analysis was 


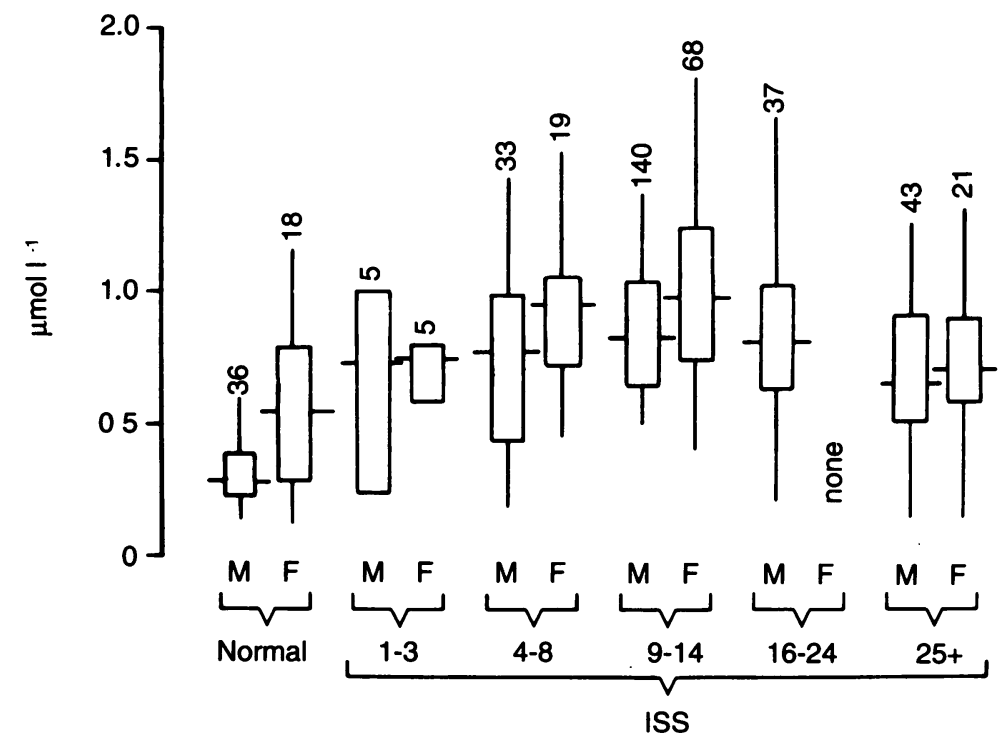

Fig. 4. Plasma cortisol concentrations in normal subjects and patients within $3 \mathrm{~h}$ of injury subdivided by sex. Conventions are as in Fig. 3.

Cortisol

carried out using GLIM (General Linear Interactive Modelling package, Royal Statistical Society).

After extraction from the database, each set of measurements was tested routinely for skewness and kurtosis ${ }^{18,19}$ assuming normal, log-normal and square-root-normal distributions. Because of the prevalence of non-normality, simple comparisons and correlations were carried out using the Wilcoxon rank sum test and Kendall's rank correlation test. These were shown to be more efficient than parametric methods ( $t$-test and simple regression), as expected ${ }^{20}$ for such non-normal data.

Multivariate regression was first carried out using the three or four independent variables that were expected to account for most of the variance of the dependent variable either from the results of simple regression or on pharmacological grounds [adrenaline], for instance, was always included. The other candidate variables were then added one at a time, and retained if the residual variance was decreased by more than $2 \%$. Finally, variables from the remaining set were removed one at a time (stepdown method) to obtain the best predictors of the dependent variable. An independent variable is said to be without effect if it accounted for less than $2 \%$ of the variance of the dependent variable.

\section{RESULTS}

\section{Preliminary observations}

Non-normality. Of the 25 sets of data large enough to test $(N>24) 10$ were skew or kurtotic $(P<0.05)$
Analgaesics, Haemaccel and unconsciousness. Plasma hormone and metabolite concentrations in patients were unaffected by analgaesics and/or Haemaccel (tested on the largest group, ISS 9-14) or by unconsciousness. The last was tested by comparing the 14 patients in the subset aged from 17 to 65 years at ISS 25 + who were unconscious for at least $1 \mathrm{~h}$ with the 22 who were not. There were no significant $(P<0.05)$ differences. Pain and awareness had, apparently, little effect.

Normal subjects. When values were affected by fasting or sex they are shown separately. [Glucose] was not significantly affected by either. Catecholamines, glucagon and vasopressin were only measured in subjects $2-5 \mathrm{~h}$ after food (fed).

Hyperglycaemia. Many patients were not hyperglycaemic: half of those up to ISS 8, were within the central $95 \%$ of the normal range, as were over a quarter of patient with ISS of 9-14 and a few at higher ISS. Values did not peak at $2 \mathrm{~h}$ after injury, as they tend to do in children with burns. ${ }^{14}$ Hyperglycaemia at ISS 1-3 was probably transient, because all values from $1.5 \mathrm{~h}$ were normal; and [glucose] rose with time at ISS $25+(P<0.005)$. Otherwise there was no time-dependence. Median values of [glucose] have been shown elsewhere to remain constant in each band from ISS 4-8 upwards for at least $8 \mathrm{~h} .{ }^{14}$

[Insulin]. Every set (Fig. 2) was roughly lognormally distributed. Median values after injury were close to those in normal non-fasted subjects and higher than in fasted normal subjects $(P<0.001$ except at ISS $25+$, when $P<0.05$ ). There was no dependence on time after injury.

[Lactate]. Values tended to be much higher at 
Glucose and

injury insulin shortly after

ISS 16+ (Fig. 3). They tended to fall with time, and at ISS 9-14 a far smaller porportion (18/103) were above normal from 1.5 to $3.0 \mathrm{~h}$ than earlier (68/147).

[NEFA]. Except at ISS 25+ values (Fig. 3) tended to be higher than in normal fed subjects $(P<0.001)$ and similar to those in normal fasted subjects. At ISS $25+$ values were intermediate. They tended to rise with time, but only significantly at ISS 9-14 $(P<0.01)$.

[Catecholamines]. Values (Fig. 3) tended to rise with ISS, as reported elsewhere, ${ }^{1,10}$ and to fall with time, but only significantly at ISS 9-14 $(P<0.05)$.

[Vasopressin]. Values were greater and more variable after injury (Fig. 3). Most were obtained at ISS 9-14.

[Glucagon]. The five values after injury were all measured within $1 \mathrm{~h}$ and at ISS $16-42$. The values were a little higher than in normal subjects and similar to others published. ${ }^{21}$

[Cortisol]. Values (Fig. 4) were higher in women than in men at ISS $4-8(P<0.05)$ and $9-14$ $(P<0.005)$, as expected, ${ }^{11}$ and rose with time after injury at ISS 4-8, 9-14 and 25+, significantly at ISS 9-14 ( $P<0.005$ in both men and women).

\section{Simple non-parametric correlations}

Theory and published correlations over wide ranges of ISS lead one to expect positive correlations between [glucose] and [adrenaline], [noradrenaline] and [vasopressin] and between [NEFA] and [noradrenaline] and negative correlations between [glucose] and [NEFA] and between [insulin] and [adrenaline]. No such correlations were found within any of the narrower ISS bands used here. [Glucose] rose with [cortisol] in females $(P<0.01$ at ISS $4-8$ and 9-14) but not in males. Age had little effect at any ISS on any metabolite. [Glucose] and [lactate] were correlated weakly with injury severity in any ISS range, more so with ISS-AIS than with ISS, though rarely significantly.

Time after food. Time after food had been recorded in 189 patients, in 26 of whom it was in the range 10-20 h (median 14 h). As expected, [NEFA] tended to rise with time after food, and [lactate], [glucose] and [insulin] to fall, but the correlation coefficients were very small, particularly so with [glucose].

\section{Multivariate analysis}

Glucose. The most normalizing transform for log[adrenaline], log[noradrenaline], log[cortisol], log[lactate] and [vasopressin]. [Insulin] was not treated as an independent variable, because it was always positively related to [glucose], i.e., [glucose] controlled [insulin], not [insulin] [glucose]. There were too few values of [glucagon] to include.

Initially, the data used were those from the 78 patients in whom [glucose], [lactate] and [adrenaline] had been measured. To increase sample sizes, they were divided into only two ISS ranges, 1-14 and 16+ (Table 1, first two columns).

The greatest dependence was on log[lactate], more at ISS $16+$ than at ISS 1-14. When log[lactate] was a variable there was no dependence on ISS-AIS or log[adrenaline], nor on any other parameter. In the absence of log[lactate] there was some, considerably smaller, dependence on ISSAIS and log[adrenaline]; but the latter had no effects independently of the former. Because [adrenaline] tended to fall with time after injury, the analysis was repeated using only data from the first hour; but the dependence on [adrenaline] was not higher, but (insignificantly) less. With log[adrenaline] and [vasopressin] as the only independent variables at ISS 1-14 the dependence on [vasopressin] was (not significantly) negative, as it was in simple regression.

Because the effects of [adrenaline] could be subsumed entirely under those of [lactate], an investigation was attempted on all patients whether or not [adrenaline] had been measured in them. The residuals were, however, very non-normally distributed. This problem was overcome by using the subset aged between 17 and 65 years.

Table 1. Percentage reduction of variance of $\sqrt{\text { [glucose] }}$ due to regression in patients with measured [adrenaline]

\begin{tabular}{lrrr}
\hline & \multicolumn{2}{c}{ All } & $\begin{array}{c}\text { Subset }^{*} \\
\text { ISS range }\end{array}$ \\
\hline$N$ & $1-14$ & $16+$ & $1-14$ \\
\hline Parameters & 46 & 32 & 24 \\
TAI $^{\dagger}$ & & & \\
ISS-AIS $^{*}$ & 0 & 2 & 24 \\
LogADR & 10 & 19 & 1 \\
LogLAC & 8 & 13 & 23 \\
LogLAC + TAI & 27 & 45 & 2 \\
ISS-AIS + logADR & 27 & 48 & 11 \\
LogLAC + ISS-AIS & 10 & 20 & 12 \\
LogLAC + LogADR & 30 & 46 & 24 \\
LogLAC + logADR + & 28 & 45 & 12 \\
ISS-AIS & 30 & 47 & 24 \\
\hline
\end{tabular}

* Subset aged 17-65 years

${ }^{\dagger}$ Abbreviations: TAI, time after injury; ADR, [adrenaline]; LAC, [lactate]. 
Members of this group were also without evidence of ethanol intake and survived at least $24 \mathrm{~h}$. Age ${ }^{16}$ and ethanol ${ }^{11}$ have known affects on [glucose] and/ or [lactate]. The effects of imminence of death were shown by comparing the 16 patients at ISS $25+$ who died within $4 \mathrm{~h}$ with the 56 who survived at least $24 \mathrm{~h}$. (Only four lived $4.1-24 \mathrm{~h}$.) In the 16 patients at ISS $25+$ the values of [lactate] $(P<0.001)$, [insulin] $(P<0.01)$ and [noradrenaline] $(P<0.01)$ were greater, those of [NEFA] $(P<0.05)$ and [cortisol] $(P<0.001)$ were less, and those of [glucose] were more variable, $1.8-19.0$ against $4.2-$ $14.8 \mathrm{mmole}^{-1}$. The $24-\mathrm{h}$ limit was used in order to leave a safety margin.

Some results of multivariate analysis are shown in Table 2. Those at ISS 1-3 are excluded, because values of [glucose] were all normal by $1.5 \mathrm{~h}$, as are a mass of near-zero correlations, such as those with sex, time after food and [cortisol]. Time after injury was often a factor, most markedly at ISS $9-14$, and for this group the results up to and from $1.5 \mathrm{~h}$ are shown separately.

At ISS 4-8 ISS-AIS was the only significant factor. At ISS 9-14 up to $1.4 \mathrm{~h}$ no combination of catecholamine, cortisol and lactate concentrations, ISS-AIS and time after injury could account for more than $4 \%$ of the variance (combination not shown in Table 2); and neither [vasopressin] nor time after food could account for as much as $2 \%$. From $1.5 \mathrm{~h}$ at this ISS, however, ISS-AIS and [lactate] had significant and partly independent effects. At ISS 16-24 [lactate] was the only factor, whereas at ISS $25+$ time after injury was equally important; but in both ranges injury severity (ISS-AIS) was a negligible factor. With log[lactate] as a factor there was no dependence on [cortisol] or time after food.

There are some discrepancies between Tables 1 and 2. The different dependency on [lactate] largely disappeared when the analysis of patients in whom [adrenaline] had been measured was confined to those who were in the subset aged between 17 and 65 years (Table 1, last column), thus excluding some elderly and one drunk patient. The higher dependence on ISS-AIS in Table 1 was wholly attributable to two high values of [glucose] in patients with high ISS-AIS from multiple limb injuries which had a greater effect on the statistics of the smaller group. Some, but not all, of the greater dependence on time after injury at ISS $25+$ shown in Table 2 than at ISS $16+$ shown in Table 1 can be explained by the combining of the two sets, ISS 16-24 and ISS 25+, in Table 1, and by a slight mismatch of times after injury.

In summary, few predictors of [glucose], even when significant, could account for more than a few per cent of the [glucose] variance. The main exceptions were [lactate] at ISS $16+$ and time after injury at ISS $25+$. There was negligible dependence on time after food. No combination of factors could explain more than about $4 \%$ of the variance at ISS $9-14$ up to $1.4 \mathrm{~h}$.

Insulin. The main group, unselected by age, etc, was used. [Insulin] depended only on [glucose] and [adrenaline], both regression coefficients being stronger in multiple than in single regression, owing to the weak positive correlation of values of [glucose] and [adrenaline] (Table 3).

The overall multiple regression coefficient for log[insulin] against $\sqrt{ }$ [glucose] was about $0.5 \pm 0.1$ which is very similar to that $(0.53)$ calculable from the results of a hyperglycaemic clamp after an overnight fast ${ }^{22}$ in which a rise of [glucose] from 5.3 to $12.2 \mathrm{mmole}^{-1}$ raised insulin from 10 to $43 \mathrm{mUI}^{-1}$. Thus the insulin response to the rise in glucose was essentially normal when allowance was made for the counter-action of adrenaline.

\begin{tabular}{|c|c|c|c|c|c|}
\hline \multirow{2}{*}{$\begin{array}{l}\text { ISS range } \\
\text { TAI }(h)^{*}\end{array}$} & \multirow[t]{2}{*}{$4-8$} & \multicolumn{2}{|c|}{$9-14$} & \multirow[t]{2}{*}{$16-24$} & \multirow[t]{2}{*}{$25+$} \\
\hline & & $0-1.4$ & $1.5-3.0$ & & \\
\hline$N$ & 59 & 89 & 57 & 22 & 32 \\
\hline \multicolumn{6}{|l|}{ Parameters } \\
\hline TAI & 0 & 1 & 0 & 3 & 20 \\
\hline ISS-AIS & 14 & 3 & 10 & 3 & 1 \\
\hline LogLAC & 0 & 2 & 12 & 51 & 22 \\
\hline $\log L A C+T A I$ & 1 & 2 & 14 & 57 & 41 \\
\hline LogLAC + ISS-AIS & 16 & 4 & 17 & 59 & 23 \\
\hline Significant $^{\dagger}$ & ISS-AIS & None & LAC & LAC & LAC,TAI \\
\hline
\end{tabular}

Table 2. Percentage of variance of $\checkmark$ [glucose] due to regression in the subset aged $17-65$ years

* Abbreviations are as in Table 1

${ }^{\dagger}$ Coefficients of regression on these parameters or their logarithms were significantly $(P<0.05)$ different from zero in multiple regression. 
Glucose and insulin shortly after injury
Because the effects of injury on [insulin] were mediated by the changes in [glucose] and [adrenaline], and these changes accounted for about a third of the variance of log[insulin] after injury, injury should have increased the variance of log[insulin] by about $50 \%$. The data yielded three estimates of the variance ratio, injured/normal, that were consistent with this view. Because most patients had not been without food for long, the first estimate is that from all the $\mathbf{4 8 9}$ patients in whom [insulin] had been measured with the 32 normal subjects $2-5 \mathrm{~h}$ after food. The ratio was 1.46 , i.e., injury increased the variance by $46 \%$. The values of the ratio when patients and normal subjects were matched for time after food were $1.49(2-5 \mathrm{~h})$ and $1.79(\geqslant 10 \mathrm{~h})$.

NEFA. Values of [NEFA] were expected to be negatively related to those of [lactate], and, by the glucose-fatty acid cycle, to [glucose] and [insulin], and positively related to [adrenaline] and [noradrenaline] owing to the lipolytic action of catecholamines and sympathetic nerve stimulation (reviewed ${ }^{2}$ ). Neither simple nor multiple regression revealed any such relationships. The only significant relationships were against expectation, negative with log[noradrenaline] at ISS $16+(P<0.01)$, and positive with log[insulin] at ISS 9-14 $(P<0.05)$.

\section{Effects of particular injuries}

Some of the variance of [glucose] might have been explained if there had been injury-specific effects on [glucose]. However, this was not the case.

From the subset aged from 17 to 65 years, 101 patients were selected whose injuries, except for minor abrasions and contusions, were confined to a single body area. Upper and lower limbs were regarded as separate areas, unlike in AIS. The ISS range was 9-11. The group with lower limb injuries was further subdivided into those with injuries confined to hip, thigh, knee, tibia and fibula, or ankle.

Values of [glucose] are shown in Fig. 5. For each compound each subset was compared with the original group minus the subset, e.g., 11 patients with fractured femur were compared with the 90 patients with other injuries. There were 40 comparisons involving [glucose], [lactate], [NEFA], [insulin] and [cortisol] with sample sizes large enough to test. Two were 'significant' $(0.05<P<0.01)$, the number most likely to occur by chance on the null hypothesis. There were too few measurements of other compounds to test. Interestingly, the effects of upper limb injuries, which have been downgraded to AIS 2 in versions of AIS later than that used (1980), were indistinguishable from those of other injuries still scored 3.

\section{DISCUSSION}

Values of [insulin] are easier to explain than those of [glucose]. Of the increase in variance of log[insulin] after injury, about $50 \%$, was wholly explicable by the increased variability of [glucose] and [adrenaline]. Mean values were similar to those in normal subjects $2-5 \mathrm{~h}$ after food. They did not vary with time after injury.

These findings are contrary to the usual view, based mostly on results given by Vitek et al. ${ }^{12}$ according to which [insulin] is raised by all but lifethreatening injuries, is very much more variable than normal, and tends to rise with time. This analysis was somewhat distorted by the use of the normal distribution for their highly skewed data and, because it is unlikely that many of their patients were fasted, by the use of fasted normal subjects as controls.

Table 3. Dependence of log[insulin] on V[glucose] and log[adrenaline].

\begin{tabular}{|c|c|c|c|c|c|c|}
\hline \multirow[t]{2}{*}{$\begin{array}{l}\text { ISS range }(\mathrm{N}) \\
\text { Simple regressi }\end{array}$} & \multicolumn{2}{|r|}{$1-14(29)$} & \multicolumn{2}{|c|}{$16+(26)$} & \multicolumn{2}{|r|}{ All (55) } \\
\hline & $\%$ var & coefft & $\%$ var & coefft & $\%$ var & coefft \\
\hline LogADR & 2 & $-0.03 \pm 0.05$ & 0 & $-0.01 \pm 0.04$ & 1 & $-0.02 \pm 0.03$ \\
\hline VGLU & 28 & $0.64 \pm 0.19$ & 29 & $0.39 \pm 0.12$ & 17 & $0.32 \pm 0.09$ \\
\hline \multicolumn{7}{|c|}{ Multiple regression } \\
\hline LogADR & $35^{*}$ & $-0.09 \pm 0.04$ & $39^{*}$ & $-0.08 \pm 0.03$ & $34^{*}$ & $-0.10 \pm 0.03$ \\
\hline VGLU & & $0.75 \pm 0.18$ & & $0.52 \pm 0.12$ & & $0.53 \pm 0.10$ \\
\hline
\end{tabular}

Abbreviations: \%var, percentage reduction in variance due to regression; coefft, regression coefficient (simple or multiple) \pm SEM; ADR, [adrenaline]; GLU, [glucose]. 


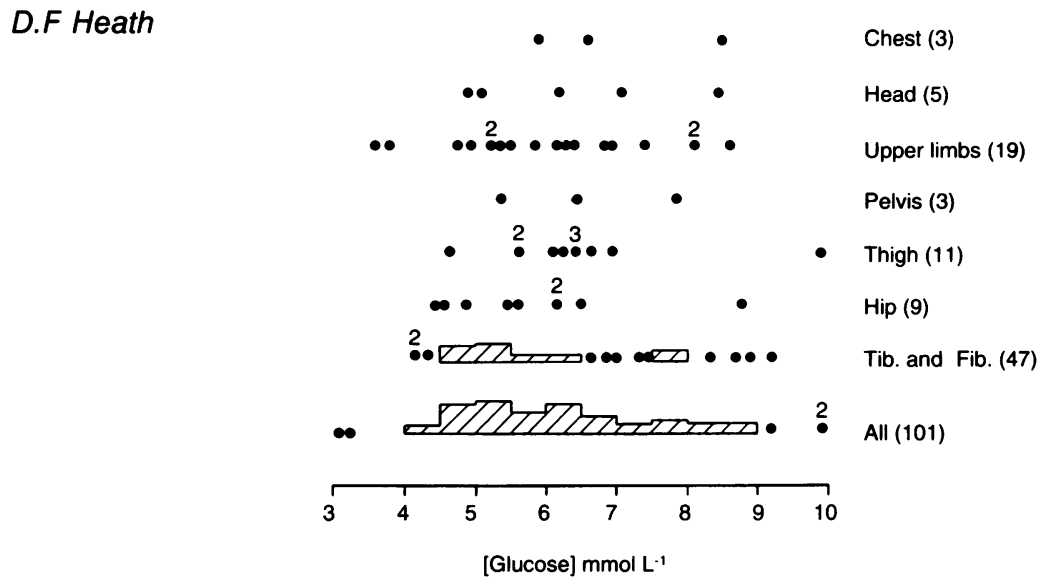

Fig. 5. Comparison of plasma glucose concentrations in a group of patients and in subgroups with particular injuries drawn from it. The groups are further described in the Main Text. The number in each group is shown in parentheses.

Recalculation of my data on their assumptions gave results very similar to those on that group of their patients who were studied within $3.1 \mathrm{~h}$ of injury on arrival at hospital. They, however, also studied another group of patients, referred from other hospitals, 3-6h after injury. These patients were more stressed and most had received treatment and their values of [insulin] were higher with variance four times as great. The impression of much greater variance and time-dependence comes from combining the two groups, although Vitek et al. ${ }^{12}$ were careful to distinguish them.

Frayn et al. ${ }^{1}$ related log[glucose], log[insulin] and log[adrenaline] in $\mathbf{4 0}$ patients, three of whom were excluded from my study. They found a clear distinction between the 20 patients in whom [adrenaline] exceeded 2 nmole $1^{-1}$ and in 20 patients in whom it was less - all six values of [insulin] above $20 \mathrm{mUI}^{-1}$ were in the patients with low [adrenaline]. I found no such difference in my larger group. Other results (Figs $2 \& 3$ ) confirm this view. Thus, the proportion of patients with [insulin] greater than $20 \mathrm{mUI}^{-1}$ was almost the same at ISS 4-8 (25/117), at which [adrenaline] rarely exceeded $2 \mathrm{nmole}^{-1}$, as at ISS $25+(14 / 72)$, at which it was rarely less. Frayn et al.'s result may well have been a chance finding, because the probability that one of two groups of 20 subjects will have no [insulin] greater than $20 \mathrm{mUI}^{-1}$ is 0.03 [calculated from the binomial theorem applied to the bulked proportion $(25+14) /(117+72)]$.

\section{Causes of hyperglycaemia}

At ISS 1-3 hyperglycaemia was only transient, and
The two main findings are the almost complete failure to account for the variability of [glucose] up to ISS 14, especially in the subset aged between 17 and 65 years, and the swamping effects of the rise in [lactate] after more severe injuries. In this group (ISS $16+$ ) the mean value of V[glucose] and its variance were higher than at ISS $4-14$, and $65 \%$ of the difference in the mean value and $85 \%$ of the difference in variance could be accounted for by the differences in the values of [lactate]. (Calculation was from the regression equations for $V$ [glucose] on log[lactate] in the two groups).

Thus, hyperglycaemia had two components, a [lactate]-independent one at all ISS, superposed on which was a [lactate]-dependent component, which was almost confined to patients with very severe or life-threatening injuries.

This picture is very unlike that given by earlier studies, referred to in the Introduction, in which [glucose], [lactate], and [adrenaline] were positively correlated over the whole range of ISS. Inspection of Figs 1 to 4 , however, shows that these correlations merely reflect the facts that the median values of [glucose], [lactate] and [adrenaline] were all higher at ISS $16+$, so that combining this group with those at lower ISS was bound to create positive correlations between all three concentrations and of each with ISS even though the correlations within the ISS 4-14 group were very poor.

The [lactate]-independent and [lactate]-dependent components of [glucose] variability are now considered in turn.

\section{The [lactate]-independent component}

For brevity only the results at ISS 9-14 are discussed.

Accepted theory failed comprehensively to account for this component of variance, especially in the first $1.4 \mathrm{~h}$. Failure to demonstrate a mechanism does not, of course, establish that it does not operate, and there were a number of factors that could not be investigated properly. These are considered briefly below, but it is hard to avoid the conclusion that there are features of the hyperglycaemic response in humans that are not understood and that are not covered by the mechanisms proposed so far.

Variability of hepatic glycogen reserves. Time after food accounted for under $2 \%$ of the variance of V[glucose]. This finding at first seems incompatible with the almost complete suppression of hyperglycaemia in the rat by $24 \mathrm{~h}$ of starvation. ${ }^{4}$ This 
Glucose and

insulin shortly after

injury period in the rat, however, is probably equivalent to at least $48 \mathrm{~h}$ of fasting in humans, and none of the patients had fasted for more than $20 \mathrm{~h}$. Liver glycogen has been measured after an overnight fast, ${ }^{23}$ and was, on average, equivalent to a plasma glucose concentration of $30 \mathrm{mmoll}^{-1}$ (assuming a glucose distribution volume of $250 \mathrm{ml} \mathrm{kg}^{-1}$ ), much higher than any measured. Thus, glycogen reserves were unlikely to have been a limiting factor in many patients.

Variability in the sympathetic stimulation of the hepatic nerve. Because hepatic nerve stimulation cannot be measured directly in patients the only evidence for sympathetic activity is the rise in [noradrenaline], which was always found, and which was considerable after more severe injuries. Correlations between [glucose] and [noradrenaline] were, however, all trivial. As is well known, plasma noradrenaline is a poor index of sympathetic stimulation, because it merely represents spill-over from synapses. A rise in [noradrenaline] is, however, good evidence of a rise in sympathetic stimulation somewhere in the body, so that, for sympathetic stimulation to account for much of the variation of [glucose], it must be assumed that the degree of sympathetic stimulation to the hepatic nerve was almost completely unrelated to that elsewhere, and was, by implication, highly specific. If so, what purpose did it fulfil? It plainly failed to produce hyperglycaemia in many recently fed patients. The hypothesis seems to be too complex to be plausible and impossible to investigate, however, it cannot be completely ruled out.

Variations in the concentrations of hormones that were not measured. There were no measurements of [angiotensin-II] and few of [glucagon]. There is some indirect evidence from studies after burn injury, which causes more hyperglycaemia than the injuries considered here, that angiotensin-II is an unlikely candidate (see Barton ${ }^{2}$ for references). The significance of the fairly slow rise of [glucagon] after injury $^{21}$ has been much debated. ${ }^{2}$ It is less than in the rat, ${ }^{8}$ but is generally held to stimulate output of glucose from the liver after the first hour. More measurements are needed (see below).

The rise in [adrenaline] was a poor index of the stimulation of glucose output by adrenaline. Because [adrenaline] fell with time, an initially close relationship between [glucose] and [adrenaline] might have been lost by the time the blood specimens were taken. The evidence is against this hypothesis. On it the [glucose]-[adrenaline] relationship should have worsened with time after injury, whereas it was slightly better $1.5-3 \mathrm{~h}$ after injury than before. Moreover, after more severe injuries, when [adrenaline] was sometimes very high, the relationship was still poor.

Variable inhibition of peripheral glucose metabolism - 'insulin resistance'. There is marked insulin resistance in the rat shortly after both dorsal scald and hind-limb ischaemia ${ }^{5,24}$ and also after injury in other species. ${ }^{25}$ In humans there is variable inhibition of glucose oxidation within $3 \mathrm{~h}$ of injury. ${ }^{26}$ In the present study the values of [NEFA] implied considerable inhibition of glucose uptake and/or metabolism. Thus, [NEFA] was at fasting levels (except at ISS $25+$, when impairment of circulation probably reduced output) even though both [glucose] and [insulin] tended to be above normal fasted, and [NEFA] was not negatively correlated with either [glucose] or [insulin]. The alternative explanation, that [NEFA] was raised by sympathetic stimulation of lipolysis, is unlikely to be true, because it was negatively, not positively correlated with [noradrenaline] even in multilinear regression with [glucose] as a second independent variable.

In principle, variations in inhibition of peripheral glucose metabolism and/or uptake could account for much of the variance of [glucose]. ${ }^{25}$ The mechanism of inhibition is, however, far from clear. That which is usually postulated, namely synergism between adrenaline, cortisol and glucagon, is based on infusion experiments on healthy adults, ${ }^{22,27,28}$ but the requisite concentrations of all three hormones are higher than those in many patients. Thus, up to ISS 14 [adrenaline] was rarely high

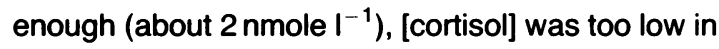
many men and some women, and [glucagon] was probably also too low. After more severe injuries [cortisol] was rarely high enough in either men or women. It is also not clear how ever-changing synergistic effects could cause [glucose] to stay nearly constant for several hours. ${ }^{14}$

A convincing test would require many more measurements on individual patients than have been carried out so far. A feasible protocol would involve sequential measurements on a group of broadly similar patients with ISS 9-14, e.g. young men with multiple lower limb injuries. The first blood specimen would be taken within $30 \mathrm{~min}$ of injury, and others at intervals not exceeding $30 \mathrm{~min}$ for at least $2 \frac{1}{2} \mathrm{~h}$. Compounds measured would include glucose, lactate, insulin, cortisol, glucagon, adrenaline, noradrenaline, vasopressin and angiotensin II. Blood gases would be measured at least once, and an assessment of blood loss would be made. 
Nutritional status, bodily habit and previous fitness would be noted.

Such a study should also reveal to what extent [glucose] does remain constant from $30 \mathrm{~min}$ in individual patients. In the main, previous work has shown constancy of group mean values.

\section{The [lactate] - dependent component}

The effects of [adrenaline] and injury severity on [glucose] were very much smaller than those of [lactate]; and most of the lactate-dependent component of [glucose] variability must be attributed to the conversion of the variable amounts of lactate to glucose. The fact that the component was practically confined to patients with very severe injuries suggests that the controlling factor was hypoxia, regional from tissue damage or general from blood loss, which increased glycolysis to lactate.

The disappearance of the effects of injury severity and [adrenaline] on [glucose] when the analysis included [lactate] does not imply that they did not exist. There are two patterns to be considered. In one, effects are mediated via [lactate]. Thus blood loss and hypoxia are likely to rise with injury severity and stimulate glycolysis, as will, in muscle, a rise in [adrenaline]. These effects on glycolysis will appear as changes in [lactate]. Because, in this mechanism, lactate is nearer to glucose production in the causal chain than ISS-AIS or adrenaline, the effects of these factors will be subsumed under those of lactate in the statistical analysis. In the other pattern, adrenaline and, from the effects of blood loss, etc., vasopressin and sympathetic stimulation may stimulate hepatic output of glucose directly, with effects that should be independent of [lactate]. Either these effects were very small in the patients studied or their apparent elimination when [lactate] was included was a statistical artefact. The latter cannot be ruled out. All these factors act in the same direction, with lactate having the greatest effect. Cases are known in least mean squares analysis in which the effects of a minor factor disappear when a major one is introduced with effects in the same direction.

\section{Injury severity scores}

Whether measured by ISS or ISS-AIS, injury severity was never a good predictor of [glucose]. ISS-AIS was only marginally better. This was surprising, because ISS-AIS includes the AIS values of all those injuries not included in the calculation of ISS, and consequently gave a much more extended scale (9-29 against 9-14 for example), with fewer tied values. Therefore, it seems unlikely that any purely anatomical description of injury severity could ever predict the metabolic response at all well.

Other injury scales based on severity of coma and estimates of impairment of circulation could not be tested, but seem unlikely to do much better, because the metabolic response was not affected by unconsciousness, and because the negligible correlation of [glucose] with [lactate] during the first $1.4 \mathrm{~h}$ at ISS 9-14 indicates that blood loss was not a major factor at this severity.

\section{GENERAL COMMENTS}

It is apparent that there are still great gaps in our understanding of hyperglycaemia after injury. Many necessary measurements have not been made, and those that have been do not provide an adequate explanation.

The matter may be of more than academic interest. The debilitating loss of protein that takes place 1 to 2 weeks following injury is known to be related to reduced insulin sensitivity. ${ }^{29}$ is there any relationship between this reduction and the early reduction? On the present analysis, these should be related, up to ISS 14, to the degree of hyperglycaemia. As far as I know this has never been investigated.

\section{ACKNOWLEDGEMENTS}

I thank all those colleagues who have allowed me to make free use of their data, even when my interpretations have conflicted with theirs, and especially John Rose, who carried out the glucagon measurements for this work, and the Salford (U.K.) Area Health Authority for financial support.

\section{REFERENCES}

1. Frayn K.N., Little R.A., Maycock P.F. \& Stoner H.B. (1985) The relationship of plasma catecholamines to acute metabolic and hormonal responses to injury in man. Circulatory Shock 16, 229-240.

2. Barton R.N. (1985) Neuroendocrine mobilization of body fuels after injury. British Medical Bulletin 41, 218-225.

3. Douglas R.G. \& Shaw J.H.F. (1989) Metabolic response to sepsis and trauma. British Journal of Surgery 76, 115-124.

4. Stoner H.B. (1958) Studies on the mechanism of 
Glucose and insulin shortly after injury shock. The quantitative aspects of glycogen metabolism after limb ischaemia in the rat. British Journal of Experimental Pathology 39, 635-651.

5. Frayn K.N. (1975) Effects of burn injury on insulin secretion and on sensitivity to insulin in the rat in vivo. European Journal of Clinical Investigation 5, 331-337.

6. Barton R.N. \& Passingham B.J. (1980) Evidence for a role of glucocorticoids in the development of insulin resistance after ischaemic limb injury in the rat. Journal of Endocrinology 86, 363-370.

7. Heath D.F. (1980) Carbohydrate metabolism after injury. The development and maintenance of hyperglycaemia. Advances of Physiological Sciences 26, 63-70.

8. Rose J.G. \& Heath D.F. (1986) The effects of stress and injury on the activity of phosphoenolpyruvate carboxykinase in the liver of the rat. Biochemical Journal 233, 239-244.

9. Davies C.L., Newman R.J., Molyneux S.G. \& Grahame-Smith D.G. (1984) The relationship between plasma catecholamines and severity of injury in man. Journal of Trauma 24, 99-105.

10. Kenney P.R., Allen-Rowlands C.F. \& Gann D.S. (1983) Glucose and osmolality as predictors of injury severity. Journal of Trauma 23, 712-719.

11. Stoner H.B., Frayn K.N., Barton R.N., Threlfall C.J. \& Little R.A. (1979) The relationships between plasma substrates and hormones and the severity of injury in 277 recently injured patients. Clinical Science 56, 563-573.

12. Vitek V., Lang D.J. \& Cowley R.A. (1979) Admission serum insulin and glucose levels in 247 accident victims. Clinica Chimica Acta 95, 93-104.

13. Copes W.S., Champion H.R., Sacco W.J., Lawnick M.M., Keast S.L. \& Bain L.W. (1988) The injury severity score revisited. Journal of Trauma 28, 69-77.

14. Childs C., Heath D.F., Little R.A. \& Brotherston M. (1990) Glucose metabolism in children during the first day after burn injury. Archives of Emergency Medicine 7, 135-146.

15. Barton R.N., Stoner H.B. \& Watson S.M. (1987) Relationships among plasma cortisol, adrenocorticotrophin, and severity of injury in recently injured patients. Journal of Trauma 27, 384-392.

16. Frayn K.N., Stoner H.B., Barton R.N., Heath D.F. \& Galasko C.S.B. (1983) Persistence of high glucose, insulin and cortisol concentrations in elderly patients with proximal femoral fracture. Age and Ageing 12, 70-76.

17. Copes W.S., Lawnick M.M., Champion H.R. \& Sacco W.J. (1988) A comparison of abbreviated injury scale 1980 and 1985 versions. Journal of Trauma 28, 78-86.

18. Geary R.C. (1936) Moments of the ratio of the mean deviation to the standard deviation for normal samples. Biometrika 25, 295-305.

19. Snedecor G.W. \& Cochran W.G. (1986) Statistical Methods, 7th ed. lowa State University Press Ames.

20. Bradley J.V. (1968) Distribution-free Statistical Tests. Prentice-Hall International Inc, London.

21. Meguid M.M., Brennan M.F., Aoki T.T., Muller W.A., Ball M.R. \& Moore F.D. (1974) Hormone-substrate interrelationships following trauma. Archives of Surgery 109, 776-783.

22. Black P.R., Brooks D.C., Bessey P.Q., Wolfe R.R. \& Wilmore D.W. (1982) Mechanism of insulin resistance following injury. Annals of Surgery 196, 420-435.

23. Nilsson L.H:Son (1973) Liver glycogen content in man in the postabsorptive state. Scandinavian Journal of Clinical and Laboratory Investigation 32, 317-323.

24. Heath D.F. \& Corney P.L. (1973) The effects of starvation, environmental temperature and injury on the rate of disposal of glucose by the rat. Biochemical Journal 136, 519-530.

25. Heath D.F. (1986) Experimental studies on energy metabolism after injury and during sepsis. In: Little R.A. \& Frayn K.N. (eds) The Scientific Basis for the Care of the Critically III, pp 95-96. Manchester University Press, Manchester.

26. Little R.A., Stoner H.B. \& Frayn K.N. (1981) Substrate oxidation shortly after accidental injury in man. Clinical Science 61, 789-791.

27. Gelfand R.A., Matthews D.E., Bier D.M. \& Sherwin R.S. (1984) Role of counter-regulatory hormones in the catabolic response to stress. Journal of Clinical Investigation 74, 2238-2248.

28. Shamoon H., Hendler R. \& Sherwin R.S. (1981) Synergistic reactions amongst antiinsulin hormones in the pathogenesis of stress hyperglycemia in humans. Journal of Clinical and Endocrinology and Metabolism $52,1235-1241$.

29. Frayn K.N. (1986) Hormonal control of metabolism in sepsis and trauma. Clinical Endocrinology 24, 577-599. 